\title{
ÉVALUATION OU SANCTION : POURQUOI ET COMMENT ENQUETTER DANS LES MUSÉES ?
}

\author{
Emmanuelle Vareille ${ }^{1}$ et Patrice de la Broise ${ }^{2}$
}

"Vous savez, je n'y connais rien. Demandez plutôt à mon mari, il saura vous répondre mieux que moi". Voilà ce qui m'a été répondu lors d'une enquête menée auprès des publics de la Grande Galerie de l'Évolution du Muséum national d'histoire naturelle de Paris tandis que je sollicitais la participation d'un visiteur ${ }^{3}$. Ce genre de réponse ne fait pas exception, même s'il est vrai que les résultats d'évaluations au musée en font généralement peu de cas. Au mieux, ces réponses sont enregistrées ou, plus exactement, comptabilisées à titre de refus ou de non-réponse. Plus rarement, elles sont questionnées jusqu'à nourrir la réflexion méthodologique.

Notre propos contribue, dans une certaine mesure, à interpréter et corriger ce type d'évitement ou de réserve manifesté à l'égard de l'enquêteur. Un évitement et/ou une réserve qui nous invitent à observer les savoirs : d'une part, en regard à un contexte d'éducation non formelle, le musée et l'exposition, et, d'autre part, en regard d'une

1 CRIPC, Université d'Avignon.

2 GERICO, Université Charles de Gaulle - Lille 3.

3 Nous faisons ici référence aux enquêtes réalisées auprès des visiteurs de la Grande Galerie de l'Évolution dans le cadre de l'Observatoire Permanent des Publics. 
expérience et non de la seule acquisition de connaissances. Pour ce faire, il convient de revenir rapidement sur les différentes méthodes d'évaluation dans les objectifs qui leur sont assignées. Un rétrospectif rapide de l'évaluation au musée indique que les méthodes d'enquête requises procèdent d'une transposition des trois principales méthodes d'investigation en usage dans les sciences sociales, à savoir l'observation, le questionnaire et l'entretien. Il faut aussi considérer l'influence des sciences de l'éducation et de ses outils méthodologiques dans les lieux d'éducation non formelle. Là encore, le questionnaire et l'entretien sont particulièrement requis.

Que prétend-t-on savoir ou obtenir en situation d'observation, de passation de questionnaire ou d'entretien?

L'observation est particulièrement utile (et utilisée) pour apprécier un comportement de visite à partir d'un parcours, de stations plus ou moins prolongées, d'interactivité entre les visiteurs et les objets, ou d'interactions, verbales et non verbales, entre les visiteurs euxmêmes. De telles observations ont été traduites en typologies de visiteurs et peuvent également prolonger une analyse formelle du dispositif d'exposition. L'évaluateur trouve ici matière à sanctionner des écarts entre l'offre d'un parcours via un dispositif (le parcours conceptuel qui suit une trame narrative) et sa ré-appropriation par le visiteur au travers d'une déambulation. Le travail fondateur de Véron et Levasseur sur l'ethnographie de l'exposition illustre l'extrapolation par laquelle on entend identifier une personnalité, des motivations et un processus cognitif au seul titre d'un relevé comportemental ${ }^{1}$.

Dans la lignée du sondage d'opinion reconnu comme relevant de méthodes quantitatives, le questionnaire d'enquête aborde, sous formes de questions fermées et/ou ouvertes, des sujets d'interrogation aussi divers que les circonstances et mobiles de visite, le déroulement de la visite, la satisfaction plus ou moins circonstanciée des visiteurs... Le questionnaire peut également être un instrument utile pour évaluer les savoirs préalables à la visite ou les acquis supposés issus de la visite. Administré à un large échantillon de personnes, le questionnaire permet d'identifier des types (types de visiteurs et de visites) et d'en mesurer les tendances. Parce qu'il constitue un dispositif simple à mettre en place qui nécessite peu de moyens humains et

1 É. Véron, M. LeVAsSeur, Ethnographie d'une exposition. L'espace, le corps et le sens, Paris, BPI - Centre Georges Pompidou, 1989. 
financiers, le questionnaire auto-administré est un mode d'enquête privilégié par nombre d'institutions muséales, en particulier pour établir un portrait sociologique de leurs usagers ${ }^{1}$.

L'entretien, libre, semi-directif ou directif, individuel ou collectif, constitue le troisième type d'investigation mobilisé dans l'évaluation muséale. Mené en amont de la recherche, il permet d'en circonscrire le cadre. On peut aussi recourir à l'entretien pour prolonger ou approfondir un objet de questionnement délimité par des recherches préalablement conduites sur la base d'observations ou de questionnaires. La qualité de l'entretien repose dans une large mesure sur la qualité de la relation qui se noue entre l'interviewé et l'interviewer. On sait depuis Palo Alto que parmi les axiomes de la communication figure, par définition, le principe de dissymétrie entre les coénonciateurs. Et tout le travail de l'enquêteur consiste à tenter de réduire dans l'empathie cette dissymétrie en faisant en sorte que les participants accèdent mutuellement au monde de références et au répertoire de l'autre.

Comment interpréter la remarque initiale à l'issue de ce bref état des lieux méthodologique ? Nul doute que celle-ci est le signe manifeste d'une appréhension à l'égard d'un questionnement identifié comme relevant d'une démarche de sanction, de contrôle. Cette appréhension trouve une justification préliminaire dans la terminologie employée : le mot évaluation ne signifie-t-il pas donner une valeur, porter un jugement. Ne parle-t-on pas aussi d'épreuves ou de tests de connaissance ? Le vocabulaire de l'évaluation muséale est pour beaucoup marqué par l'héritage de l'univers scolaire et des sciences de l'éducation. Nous faisons ici référence à la typologie de Screven, qui, s'appuyant sur le modèle scolaire proposé par son homonyme Scriven, distingue l'évaluation préalable, celle qui précède la réalisation de l'exposition, l'évaluation formative qui accompagne la mise en exposition et l'évaluation formative qui a lieu après l'ouverture de l'exposition au public ${ }^{2}$. Mais le musée n'est pas l'école. Cette simple remarque suffit à s'interroger sur la pertinence et les

1 Pour relayer le musée dans la connaissance de ses publics, il existe différentes structures, dont l'Observatoire Permanent des Publics (OPP) qui offre des prestations centrées sur l'audience des musées et, plus largement sur la place du musée dans une pratique culturelle.

2 C. G. SCREVEN, "Uses of evaluation before, during and after exhibits design", ILVS Review : A journal of visitor behaviour, vol. 1, $\mathrm{n}^{\circ} 2,1990, \mathrm{pp} .36-66$. 
conséquences d'une transposition des méthodes de l'évaluation scolaire à l'univers muséal. À la différence de l'institution certificative, le musée n'a pas pour fonction le contrôle et la validation des acquis. La principale question reste en fait de savoir ce que l'évaluation sanctionne. Le dispositif et, à travers lui, le concepteur ou le visiteur? L'évaluateur doit veiller à lever cette ambiguïté en rappelant régulièrement les objectifs de l'évaluation. À ce titre, le statut de l'évaluateur est primordial : qui représente-t-il ? Est-il partie prenante de l'institution muséale? Nous savons par expérience qu'il est difficile d'être à la fois juge et partie.

Il faut en outre considérer que nous sommes dans un lieu de culture, qui plus est un lieu hautement symbolique, souvent considéré sur le mode de l'avoir autant qu'en termes d'expérience sensorielle, affective ou sociale. Du côté du visiteur, la visite d'un musée est souvent rapportée à un actif. N'entend-t-on pas dire "J'ai fait le Louvre", sous-entendu, Le Louvre compte parmi mes propres souvenirs. Du côté de l'institution commanditaire de l'évaluation, les effets de la visite -et donc la qualité d'une exposition- sont souvent mesurés à l'aune de sa portée éducative. Il peut s'ensuivre une sorte de démission de la part de visiteurs se considérant non compétents, la notion de compétence étant à considérer non seulement du point de vue encyclopédique, mais aussi linguistique et générique.

Cette appréhension fait aussi écho à la situation même de l'évaluation. Le visiteur et l'évaluateur sont inscrits dans une situation de communication dissymétrique dans laquelle les savoirs formels sont généralement ceux que questionne l'enquêteur et dont l'enquêté se sent justifiable. Il y a en quelque sorte celui qui est supposé savoir et qui questionne et celui qui est supposé savoir et qui répond. La relation dans laquelle l'un est en droit et en position d'interroger l'autre crée par elle-même une situation d'inégalité, et pour tout dire d'examen. Nous voulons pour preuve de cette relation dissymétrique le phénomène bien connu des réponses de complaisance ou réponses nobles, celles que le visiteur subodore comme correspondant aux "bonnes réponses", parce que les plus acceptables socialement ou les plus susceptibles de plaire à l'enquêteur.

Peux-t-on éviter cela ? Si oui, comment? En privilégiant d'autres objets de questionnement -c'est-à-dire en mettant l'accent sur l'expérience du visiteur- et/ou en privilégiant d'autres formes de questionnement. Nous donnons ici deux exemples de protocoles 
d'enquêtes, expérimentés auprès des visiteurs de la Grande Galerie de l'Évolution qui, tous deux, participent de cette tentative.

L'entretien itinérant, tel que nous l'avons baptisé, consiste en quelque sorte en une observation participante assortie d'un questionnement du visiteur. Cet entretien se déroule au cours de la visite : c'est le cheminement du visiteur qui en détermine la structure et la cadence. Cette méthode, en même temps qu'elle réduit le décalage entre le moment où a lieu la visite et celui où elle est prétexte à un questionnement, met davantage l'accent sur les procédures d'une appropriation de l'exposition par le visiteur que sur les seuls effets de la visite. Reste que dans cette situation d'enquête, le visiteur interrogé est toujours enclin à faire œuvre de performance. Cela se traduit notamment à travers les parcours de visite. La plupart des visiteurs manifestent leur souci d'inspecter l'exposition dans ses moindres recoins. "Est-ce qu'on a fait le tour ?", s'inquiètent les uns, "Qu'est-ce qu'il nous reste à voir ?", demandent les autres. À en juger par l'enchaînement des éléments d'exposition, qui n'est pas sans rappeler la stratégie du visiteur fourmi, tout se passe comme si le visiteur, fort de son engagement, s'efforçait d'être le plus rigoureux possible pour garantir un rendement optimal de sa visite.

L'approche des interactions dans l'exposition ne se limite évidemment pas à l'observation participante de pratiques muséales, fût-elle enrichie par un questionnement direct du visiteur. L'interaction met en jeu des mécanismes, des logiques de reconnaissance (assistés et / ou contingentés) qui ne peuvent être verbalisés uniquement sous la forme de questions-réponses en référence directe et explicite avec un contenu et une forme muséographique. Le chercheur peut envisager des formules de décentrage susceptibles, en travaillant à la périphérie de l'objet dont il est question, de questionner plus adroitement peut-être et plus sensiblement sûrement l'interaction au musée.

Prenons pour exemple une étude du souvenir de la visite de la Grande galerie de l'évolution passées huit semaines. Pour une part, cette étude mobilise un mode de questionnement inhabituel qui repose sur le principe du jeu ou portrait chinois. Ce jeu a pour principe initial de deviner le nom d'une personnalité à partir d'informations diverses fournies par le meneur de jeu en réponse à des questions de forme "si c'était un arbre...", "si c'était un animal..."... Indépendamment de l'interprétation des résultats par le chercheur, les répondants sont systématiquement invités à commenter leurs propres choix. 
Ce procédé a pour dessein de dépasser le cadre strict de l'acquisition de connaissances. Il dissuade le visiteur interrogé de favoriser des rappels précis mobilisés dans le cadre des objectifs de sa visite et l'incite, au contraire, à demeurer le plus possible dans l'instantané. Le portrait chinois présente en outre l'avantage de provoquer un type de paraphrase particulier en faisant correspondre, à une image (celle de la galerie), une autre image, celle qu'en donne le visiteur à travers différentes associations. Concrètement parlant, le portrait chinois présente deux avantages supplémentaires. Par son côté ludique, il libère la parole et dédramatise la situation qui, sans cela, pourrait être perçue comme un examen d'évaluation du visiteur et des qualités de sa visite. Ensuite, par la familiarité de sa forme, le portrait chinois simplifie la procédure de questionnement et délimite le mode et le champ d'investigation choisi par le visiteur.

Les méthodes brièvement présentées ici ne sont pas innovantes en elles-mêmes, mais par leur transposition dans des lieux où elles ne sont pas privilégiées, notamment parce qu'elles ne sont pas là en sanction d'un dispositif ou d'un public, qu'elles ne stigmatisent pas des performances ou des compétences.

Nous ne sommes pas sûrs d'être là dans le cadre des savoirs informels au sens strictement cognitif du terme. Quand bien même s'agirait-il d'approcher des savoirs informels, il faut considérer que la délectation participe de la fonction éducative du musée. Et parce que l'acquisition de savoirs ne se réduit pas à une chaîne cognitive, il faut probablement questionner le plaisir et l'émotion rencontrés au cours de la visite. Les méthodes expérimentées dans le cadre d'une recherche doctorale privilégient en l'occurrence les conditions d'intimité, de jeu et de projection. Il s'agit moins de savoir que d'expérience. Ce n'est pas parce qu'on est dans l'expérience, qu'on doit systématiquement privilégier le récit de l'expérience. Ce qui nous intéresse, ce ne sont pas tant les savoirs que leurs processus, les conditions et les circonstances dans lesquels ils émergent. 